\title{
Symmetries of Taub-NUT Dual Metrics
}

\author{
Dumitru Băleanu円, Sorin Codoban円 \\ Joint Institute for Nuclear Research \\ Bogoliubov Laboratory of Theoretical Physics, \\ 141980 Dubna, Moscow Region, Russia
}

\begin{abstract}
In this paper we study the symmetries of the dual Taub-NUT metrics. Generic and non-generic symmetries of dual Taub-NUT metrics are investigated. The existence of the Runge-Lenz type symmetry is analyzed for dual Taub-NUT metrics. We find that in some cases the symmetries of the dual metrics are the same with the symmetries of Taub-NUT metric.
\end{abstract}

\footnotetext{
${ }^{1}$ after 1st March 1999 at Institute for Space Sciences, P.O.BOX, MG-36, R 76900, Bucharest-Măgurele,Romania, e-mail:baleanu@roifa.ifa.ro

${ }^{2}$ E-mail address: baleanu@thsun1.jinr.ru

${ }^{3}$ E-mail address: codoban@thsun1.jinr.ru
} 


\section{Introduction}

In a geometrical setting, symmetries are connected with isometries associated with Killing vectors and, more generally, Killing tensors on the configurations space of the system. An example is the motion of a point particle in a space with isometries [1], which is a physicist's way of studying the geodesic structure of a manifold. In [1] such studies were extended to spinning space-times described by supersymmetric extensions of the geodesic motion, and in [2] it was shown that this can give rise to interesting new types of supersymmetry as well.

The geometric duality between a metric $g^{\mu \nu}$ and its non-degenerate Killing tensor $K^{\mu \nu}$ was discussed in [3]. The relation was generalized to spinning spaces, but only at the expense of introducing torsion. The physical interpretation of the dual metrics was not clarified [3]. The geometrical interpretation of Killing tensors was investigated in 4 .

Recently the structural equations for a Killing tensor of order two was investigated and the geometric duality between $g_{\mu \nu}$ and a non-degenerate Killing tensor $K_{\mu \nu}$ was analyzed in [5].

An interesting example of an Einstein's metric which admits Killing-Yano tensors is Taub-NUT metric. Taub-NUT metric is involved in many modern studies in physics. For example the Kaluza-Klein monopole of Gross and Perry [6] and of Sorkin [7] was obtained by embedding the Taub-NUT gravitational instanton into five-dimensional Kaluza-Klein theory. Remarkably, the same object has re-emerged in the study of monopole scattering. In the long distance limit, neglecting radiation, the relative motion of the BPS monopoles is described by the geodesics of this space [8] [9]. The dynamics of well-separated monopoles is completely soluble and has a Kepler type symmetry [10, 11, 12, 13].

The geodesic motion of pseudo-classical spinning particles in Euclidian Taub-NUT were analyzed in [14] and the symmetries of extended Taub-NUT metrics recently were studied in [15, 16, 17]. Taub-NUT metric admits four Killing-Yano tensors which generate four non-degenerate Killing tensors [3]. On the other hand for a given manifold $g_{\mu \nu}$ which admits a non-degenerate Killing tensor $K_{\mu \nu}$ two types of dual metrics exist [5]. An interesting question is to investigate the connection between the symmetries of dual Taub-NUT metrics and the symmetries of Taub-NUT metric.

For these reasons the symmetries of the Taub-NUT dual metrics will be analyzed. The aim of this paper is to investigate the generic and non-generic symmetries corresponding to geodesic motion of pseudo-classical spinning particles on the Taub-NUT dual metrics. We will investigate the existence of the Runge-Lenz symmetry for the dual metrics.

The organization of the paper is as follows. In Section 2 the geometric duality is presented. In Section 3 we investigate the symmetries corresponding to Taub-NUT dual metrics and we construct the spinning space. In Section 4 we present our conclusions. 
In Appendix 1 we write down Christoffel symbols and the scalar curvature for two interesting dual metrics. The calculus for all Taub-NUT dual metrics were done, but due to their huge and complicated expressions we cannot write them out in this paper. In Appendix 2 we present two plots of scalar curvature of two interesting dual metrics.

\section{Geometric Duality}

The equation of motion of a particle on a geodesic is derived from the action

$$
S=\int d \tau \frac{1}{2} g_{\mu \nu} \dot{x^{\mu}} \dot{x}^{\nu}
$$

The Hamiltonian has the form $H=\frac{1}{2} g_{\mu \nu} p^{\mu} p^{\nu}$ where the Poisson brackets are $\left\{x_{\mu}, p^{\nu}\right\}=\delta_{\mu}^{\nu}$.

Let us suppose that the metric $g_{\mu \nu}$ admits a Killing tensor field $K_{\mu \nu}$. A Killing tensor is a symmetric tensor which satisfies the following relation:

$$
D_{\lambda} K_{\mu \nu}+D_{\mu} K_{\nu \lambda}+D_{\nu} K_{\lambda \mu}=0
$$

where $D_{\mu}$ denote covariant derivatives. From the covariant components $K_{\mu \nu}$ of the Killing tensor one can construct a constant of motion $K=\frac{1}{2} K_{\mu \nu} p^{\mu} p^{\nu}$. It can be easy verified that $\{H, K\}=0$.

The formal similarity between the constants of motion $\mathrm{H}$ and $\mathrm{K}$, and the symmetrical nature of the condition implying the existence of the Killing tensor amount to a reciprocal relation between two different models:the model with Hamiltonian $\mathrm{H}$ and constant of motion $\mathrm{K}$, and a model with constant of motion $\mathrm{H}$ and Hamiltonian K.The relation between the two models has a geometrical interpretation: it implies that if $K_{\mu \nu}$ are the contravariant components of a Killing tensor with respect to the metric $g_{\mu \nu}$, then $g_{\mu \nu}$ must represent a Killing tensor with respect to the metric defined by $K_{\mu \nu}$. When $K_{\mu \nu}$ has an inverse we interpret it as the metric of another space and we can define the associated Riemann-Christoffel connection $\hat{\Gamma}_{\mu \nu}^{\lambda}$ as usual through the metric postulate $\hat{D}_{\lambda} K_{\mu \nu}=0$. Here $\hat{D}$ represents the covariant derivative with respect to $K_{\mu \nu}$.

This reciprocal relation between the metric structure of pairs of spaces constitutes a duality relation: performing the operation of mapping a Killing tensor to a metric twice leads back to the original theory.

The geometric duality between $g_{\mu \nu}$ and a Killing tensor $K_{\mu \nu}$ was analyzed in [5].In this case Killing's vectors equations in the dual space have the following form [5

$$
D_{\mu} \hat{\chi}_{\nu}+D_{\nu} \hat{\chi}_{\mu}+2 K^{\delta \sigma}\left(D_{\delta} K_{\mu \nu}\right) \hat{\chi}_{\sigma}=0
$$

Here $\hat{\chi}_{\sigma}$ are Killing vectors in dual spaces.

Let us suppose that metric $g_{\mu \nu}$ admits a Killing-Yano tensor $f_{\mu \nu}$. A KillingYano tensor is an antisymmetric tensor [2] which satisfies $D_{\mu} f_{\nu \lambda}+D_{\nu} f_{\mu \lambda}=0$ 
The corresponding Killing-Yano equations in the dual space has the form [5

$$
D_{\mu} \hat{f}_{\nu \lambda}+D_{\nu} \hat{f}_{\mu \lambda}+\hat{f}_{\nu}^{\delta} D_{\delta} K_{\mu \lambda}+2 \hat{f}_{\lambda}^{\sigma} D_{\sigma} K_{\nu \mu}+\hat{f}_{\mu}^{\delta} D_{\delta} K_{\nu \lambda}=0
$$

where $\hat{f}_{\mu \nu}$ is a Killing-Yano tensor on the dual manifold. D represents the covariant derivative with respect to $g_{\mu \nu}$.

\section{Symmetries of the dual metrics for the self- dual Euclidean Taub-NUT metric}

The four-dimensional Taub-NUT metric depends on a parameter $m$ which can be positive or negative, depending on the application; for $m>0$ it represents a nonsingular solution of the self-dual Euclidean equation and as such is interpreted as a gravitational instanton. The standard form of the line element is

$$
\begin{aligned}
d s^{2}= & \left(1+\frac{2 m}{r}\right)\left(d r^{2}+r^{2} d \theta^{2}+r^{2} \sin ^{2} \theta d \varphi^{2}\right) \\
& +\frac{4 m^{2}}{1+2 m / r}(d \psi+\cos \theta d \varphi)^{2}
\end{aligned}
$$

The Killing vectors for the metric (5) have the following form:

$$
D^{(\alpha)}=R^{(\alpha) \mu} \partial_{\mu}, \quad \alpha=1, \cdots, 4
$$

where

$$
\begin{aligned}
D^{(1)} & =\frac{\partial}{\partial \psi} \\
D^{(2)} & =-\frac{\partial}{\partial \varphi} \\
D^{(3)} & =\sin \varphi \frac{\partial}{\partial \theta}+\cos \varphi \cot \theta \frac{\partial}{\partial \varphi}-\frac{\cos \varphi}{\sin \theta} \frac{\partial}{\partial \psi} \\
D^{(4)} & =-\cos \varphi \frac{\partial}{\partial \theta}+\sin \varphi \cot \theta \frac{\partial}{\partial \varphi}-\frac{\sin \varphi}{\sin \theta} \frac{\partial}{\partial \psi}
\end{aligned}
$$

The metric (5) admits four Killing-Yano tensors [18]. Three of these, denoted by $f_{i}$ are special because they are covariant constant. In the two-form notation the explicit expressions are

$$
f_{i}=4 m(d \psi+\cos \theta d \varphi) d x_{i}-\epsilon_{i j k}\left(1+\frac{2 m}{r}\right) d x_{j} \wedge d x_{k}
$$

where the $d x_{i}$ are standard expressions in terms of the 3 -dimensional spherical co-ordinates $(r, \theta, \varphi)$. The fourth Killing-Yano tensor has the following form

$$
Y=4 m(d \psi+\cos \theta d \varphi) \wedge d r+4 r(r+m)\left(1+\frac{r}{2 m}\right) \sin \theta d \theta \wedge d \varphi
$$


Now we would like to investigate the symmetries of the dual Taub-NUT metrics.

We will construct firstly the Taub-NUT dual metrics using the geometric duality between $g_{\mu \nu}$ and $K_{\mu \nu}$ [5]. We know that when a manifold M admits a Killing-Yano tensor $f_{\mu \nu}$ we can construct a corresponding Killing tensor 14] $K_{\mu \nu}$ as

$$
K_{\mu \nu}=f_{\mu}^{\lambda} f_{\nu \lambda}
$$

Using (13) and (12) the line element for the dual metric becomes

$$
\begin{aligned}
d k^{2}= & \left(1+\frac{2 m}{r}\right)\left(d r^{2}+\frac{r^{2}}{m^{2}}(r+m)^{2}\left(d \theta^{2}+\sin ^{2} \theta d \varphi^{2}\right)\right) \\
& +\frac{4 m^{2}}{1+2 m / r}(d \psi+\cos \theta d \varphi)^{2} .
\end{aligned}
$$

The Taub-NUT metric admits three more second-rank Killing tensors of the form [18]

$$
K_{\mu \nu}^{(i)}=f_{\mu \lambda}^{(i)} Y_{\nu}^{\lambda}+f_{\nu \lambda}^{(i)} Y_{\mu}^{\lambda}
$$

Here $f_{\mu \nu}^{i}$ and $Y_{\mu \nu}$ are given by (11) and(12). They form a conserved vector of Runge-Lenz type. Using (15) the line element for the dual metrics becomes

$$
\begin{aligned}
d k_{(i)}^{2}= & -\frac{2}{m}\left(1+\frac{2 m}{r}\right)\left(1+\frac{m}{r}\right) r_{i}\left(d r^{2}+r^{2} d \theta^{2}+r^{2} \sin ^{2} \theta d \varphi^{2}\right)+ \\
& \frac{8 m^{2}}{r(1+2 m / r)} r_{i}(d \psi+\cos \theta d \varphi)^{2}+\frac{2 r}{m}\left(1+\frac{2 m}{r}\right)^{2} d r d r_{i} \\
& +4\left(1+\frac{2 m}{r}\right)(\mathbf{r} \times d \mathbf{r})_{i}(d \psi+\cos \theta d \varphi)
\end{aligned}
$$

We found that the dual metric (14) has the same Killing vectors like TaubNUT metric (5) because relation (3) is identically satisfied.

For $i=3$ the corresponding metric from (16) admits two Killing vectors (17) and (8) but for $i=1,2$ we found from (3) only one Killing vector (7).

Because the Weyl tensor of (14) has non-vanishing components (e.g $C_{1234}=2\left(m^{2} r \sin (\theta)(2 r+3 m) /(r+2 m)^{2}(r+m)\right)$, the metric is not conformally flat. We have obtained the same result for (16) but the expressions are too long to be provided here.

Now we would like to investigate the Killing-Yano tensors of order two for the dual metrics (14,16).

We have six independent components of Killing-Yano tensor $f_{\mu \nu}$ and 24 independent equations (41).

Replacing $D_{\mu} f_{\lambda \gamma}=\partial_{\mu} f_{\lambda \gamma}-f_{\delta \gamma} \Gamma_{\lambda \mu}^{\delta}-f_{\lambda \delta} \Gamma_{\mu \gamma}^{\delta}$ in (⿻) and using the corresponding expressions of Christoffel's symbols for (14) we get a set of Killing-Yano equations. We found that (4) has no solution, therefore the dual metrics (14) and (16) have no extra symmetry of Runge-Lenz type. 
In [3] using geometric duality between $g^{\mu \nu}$ and $K^{\mu \nu}$ four dual Tab-NUT metrics were found. The inverse matrix of the covariant form from (14) give us the dual line element

$$
\begin{aligned}
d \tilde{s}^{2}= & \left(1+\frac{2 m}{r}\right)\left(d r^{2}+\frac{m^{2} r^{2}}{(r+m)^{2}}\left(d \theta^{2}+\sin ^{2} \theta d \varphi^{2}\right)\right) \\
& +\frac{4 m^{2}}{1+2 m / r}(d \psi+\cos \theta d \varphi)^{2} .
\end{aligned}
$$

and the dual metrics corresponding to Runge-Lenz vector have the following form (for more details see [3]).

$$
\begin{aligned}
d \tilde{s}_{(i)}^{2}= & \frac{-1}{r_{i}^{2}-(r+2 m)^{2}}\left\{-\frac{2 m^{2}}{r}\left(1+\frac{2 m}{r}\right) r_{i}\left(d r^{2}+r^{2} d \theta^{2}+r^{2} \sin ^{2} \theta d \varphi^{2}\right)\right. \\
& +\frac{8 m^{3}(1+m / r)}{(1+2 m / r)} r_{i}(d \psi+\cos \theta d \varphi)^{2}+2 m r\left(1+\frac{2 m}{r}\right)^{2} d r d r_{i} \\
& \left.+4 m^{2}\left(1+\frac{2 m}{r}\right)(\mathbf{r} \times d \mathbf{r})_{i}(d \psi+\cos \theta d \varphi)\right\}
\end{aligned}
$$

Next step is to investigate the symmetries of the metrics (17, 18).

If we make the transformation to a new variable $\mathrm{u}$ as $u=r e^{\frac{r}{m}}$ the line element (17) becomes

$$
d \tilde{s}^{2}=F(u)\left(d u^{2}+u^{2}\left(d \theta^{2}+\sin ^{2} \theta d \varphi^{2}\right)\right)+G(u)\left(d \psi+\cos \theta d \varphi^{2}\right)
$$

where $F(u)$ and $G(u)$ are given by $F\left(r e^{\frac{r}{m}}\right)=\frac{e^{-\frac{2 r}{m}}}{\left(\frac{1}{m}+\frac{1}{r}\right)^{2} r^{2}}\left(1+\frac{2 m}{r}\right)$,

$G\left(r e^{\frac{r}{m}}\right)=\frac{4 m^{2}}{1+\frac{2 m}{r}}$. The metric (19) is a particular form of extended Taub-NUT metric presented in [15, 16]. On the other hand, in the case of geometric duality between $g^{\mu \nu}$ and $K^{\mu \nu}$ the equations (3) and (4) are not valid (for more details see [5]). Because of this the symmetries of the metrics (17, 18) will be investigated using (see [2, 3]) the Killing vectors equations

$$
\hat{D}_{\mu} \hat{\chi}_{\nu}+\hat{D}_{\nu} \hat{\chi}_{\mu}=0
$$

and the Killing-Yano equations

$$
\{\mu \nu \lambda\}=\hat{D}_{\mu} \hat{f}_{\nu \lambda}+\hat{D}_{\nu} \hat{f}_{\mu \lambda}=0
$$

Solving (20) we found that the dual metric (17) admits the same Killing vectors as metric (5).This result is in agreement with those from 15. For $i=3$, (20) give us for the corresponding metric in (18) two Killing vectors (7) and (8). In the case $i=1,2$ from (18) we found only one Killing vector (7) for the corresponding metrics.

Now we investigate if the dual metrics (17, 18) admits Killing-Yano tensors of order two. Our strategy is quite straightforward in this case. We simply 
write down all the components of the equation (21) explicitly. The total number of components of $f_{\mu \nu}$ is six, while the number of independent equations for eq.(21) is 24 .

$$
\begin{array}{ll}
\{r r \theta\}=0 & ,\{r r \varphi\}=0,\{r r \psi\}=0,\{\theta \theta r\}=0,\{\theta \theta \varphi\}=0 \\
\{\theta \theta \psi\}=0 & ,\{\varphi \varphi r\}=0,\{\psi \psi r\}=0,\{\psi \psi \theta\}=0,\{\psi \psi \varphi\}=0 \\
\{r \theta \varphi\}=0 & ,\{r \theta \psi\}=0,\{r \varphi \theta\}=0,\{r \varphi \psi\}=0,\{r \psi \theta\}=0 \\
\{r \psi \varphi\}=0 & ,\{\theta \varphi r\}=0,\{\theta \psi r\}=0,\{\varphi \theta \psi\}=0,\{\varphi \varphi \theta\}=0 \\
\{\psi \theta \varphi\}=0 & ,\{\psi \varphi r\}=0,\{\psi \varphi \theta\}=0,\{\varphi \varphi \psi\}=0
\end{array}
$$

Solving (22) we found no solution for the metrics (17) and (18). Then the dual metrics (17,18) have no Killing-Yano tensors and Runge-Lenz type symmetry. The Weyl tensor has non-vanishing components for (17)(e.g. $C_{1234}=$ $\left.-2 m^{3} r \sin (\theta) /(r+m)(r+2 m)^{2}\right)$ and then the metric in not conformally flat. Metrics (18) are not conformally flat because they have non-vanishing Weyl tensor components, but all expressions are too long to be written here.

\subsection{Generic and non-generic symmetries}

An action for the geodesic of spinning space is:

$$
S=\int_{a}^{b} d \tau\left(\frac{1}{2} g_{\mu \nu}(x) \dot{x}^{\mu} \dot{x}^{\nu}+\frac{i}{2} g_{\mu \nu}(x) \psi^{\mu} \frac{D \psi^{\nu}}{D \tau}\right)
$$

The overdot denotes an ordinary proper-time derivative $d / d \tau$, whilst the covariant derivative of a Grassmann variable $\psi^{\mu}$ is defined by $\frac{D \psi^{\mu}}{D \tau}=\dot{\psi}^{\mu}+\dot{x}^{\lambda} \Gamma_{\lambda \nu}^{\mu} \psi^{\nu}$ . In general, the symmetries of a spinning-particle model can be divided into two classes. First, there are conserved quantities which exist in any theory and these are called generic constants of motion . It was shown that for a spinning particle model defined by the action (23) there are four generic symmetries [14.

1. Proper -time translations and the corresponding constant of motion are given by the Hamiltonian:

$$
H=\frac{1}{2} g^{\mu \nu} \Pi_{\mu} \Pi_{\nu}
$$

2. Supersymmetry generated by the supercharge

$$
Q=\Pi_{\mu} \psi^{\mu}
$$


3. Chiral symmetry generated by the chiral charge

$$
\Gamma_{*}=\frac{1}{4 !} \sqrt{-g} \epsilon_{\mu \nu \lambda \sigma} \psi^{\mu} \psi^{\nu} \psi^{\lambda} \psi^{\sigma}
$$

4. Dual supersymmetry, generated by the dual supercharge

$$
Q^{*}=\frac{1}{3 !} \sqrt{-g} \epsilon_{\mu \nu \lambda \sigma} \Pi^{\mu} \psi^{\nu} \psi^{\lambda} \psi^{\sigma} .
$$

Here $\Pi_{\mu}=g_{\mu \nu} \dot{x}^{\mu}$ represents the covariant momentum.

The second kind of conserved quantities, called non-generic, depend on the explicit form of the metric $g_{\mu \nu}(x)$. Non-generic symmetries are associated with the existence of Killing- Yano tensors on a given manifold. The existence of a Killing-Yano tensor $f_{\mu \nu}$ of the bosonic manifold is equivalent to the existence of a supersymmetry for the spinning particle with supercharge

$Q_{f}=f_{a}^{\mu} \Pi_{\mu} \psi^{a}-\frac{1}{3} i H_{a b c} \psi^{a} \psi^{b} \psi^{c}$ satisfies $\left\{Q, Q_{f}\right\}=0$, where $H_{\mu \nu \lambda}=D_{\lambda} f_{\mu \nu}$.

For the metric (14) we found the generic symmetries (24 27) in the following form

$$
\begin{aligned}
& H=-\frac{1}{2} \frac{\left(-r^{2} m^{2}-4 r m^{3}-4 m^{4}\right) \dot{r}^{2}}{m^{2}(r+2 m) r} \\
& -\frac{1}{2} \frac{\left(-6 r^{5} m-12 r^{3} m^{3}-13 r^{4} m^{2}-r^{6}-4 m^{4} r^{2}\right) \dot{\theta}^{2}}{m^{2}(r+2 m) r} \\
& -\frac{1}{2}\left(-13 r^{4} m^{2} \sin (\theta)^{2}-6 r^{5} m \sin (\theta)^{2}-r^{6} \sin (\theta)^{2}-4 m^{4} r^{2}\right. \\
& \left.-12 r^{3} m^{3} \sin (\theta)^{2}\right) \dot{\phi}^{2} /\left(m^{2}(r+2 m) r\right) \\
& +4 \frac{m^{2} \cos (\theta) r \dot{\phi} \dot{\psi}}{r+2 m}+2 \frac{r m^{2} \dot{\psi}^{2}}{r+2 m} \\
& Q=\left(1+2 \frac{m}{r}\right) \dot{r} \psi^{r}+\frac{r^{2}\left(1+2 \frac{m}{r}\right)(r+m)^{2} \dot{\theta} \psi^{\theta}}{m^{2}}+ \\
& \left(\left(\frac{r^{2}(r+m)^{2}\left(1+2 \frac{m}{r}\right) \sin (\theta)^{2}}{m^{2}}+4 \frac{m^{2} \cos (\theta)^{2}}{1+2 \frac{m}{r}}\right) \dot{\phi}+4 \frac{m^{2} \cos (\theta) \dot{\psi}}{1+2 \frac{m}{r}}\right) \psi^{\varphi} \\
& +\left(4 \frac{m^{2} \cos (\theta) \dot{\phi}}{1+2 \frac{m}{r}}+4 \frac{m^{2} \dot{\psi}}{1+2 \frac{m}{r}}\right) \psi^{\psi} \\
& \Gamma_{*}=\frac{1}{12 m}(r+2 m) r(r+m)^{2} \sin (\theta)^{2} \psi^{r} \psi^{\theta} \psi^{\varphi} \psi^{\psi}
\end{aligned}
$$




$$
\begin{aligned}
Q^{*}= & \frac{(r+2 m) r(r+m)^{2} \sin (\theta)^{2}}{3 m}\left(\dot{r} \psi^{\theta} \psi^{\varphi} \psi^{\psi}-\dot{\theta} \psi^{r} \psi^{\varphi} \psi^{\psi}\right. \\
& \left.+\dot{\varphi} \psi^{r} \psi^{\theta} \psi^{\psi}-\dot{\psi} \psi^{r} \psi^{\theta} \psi^{\varphi}\right)
\end{aligned}
$$

Because the determinants of all dual metrics $(10,17,18)$, do not vanish we have similar expressions, in corresponding cases, for the generic symmetries (24 27).

As it was shown above the dual metrics have no Killing-Yano tensors, and because of this we have no non-generic symmetries for metrics (14, 16, 17, 18).

\section{Conclusions}

Recently geometric duality was analyzed for a metric which admits a nondegenerate Killing tensor of order two [3, 5]. For a given manifold which admits a non-degenerate Killing tensor of order two, geometric duality give us two types of dual metrics [5]. An interesting example arises when the manifold admits Killing-Yano tensors because they generate Killing tensors.

In this paper the symmetries of the dual Taub-NUT metrics were investigated. Taub-NUT metric admits four Killing-Yano tensors of order two and we have four corresponding dual metrics. We have obtained that the number of Killing vectors of the dual Taub-NUT metrics depend drastically on their particular form. We found that metrics (14, 177) have the same Killing vectors as the Taub-NUT metric (5), the corresponding dual metrics for $i=3$ in (16,18) have two Killing vectors (7,8), and for $i=1,2$ we get only one Killing vector $(\overline{7})$. We have obtained, by solving Killing-Yano equations, that all dual Taub-NUT metrics do not have Killing-Yano tensors. This means that all dual metrics do no admit extra symmetries of Runge-Lenz type.

The scalar curvature of Taub-NUT metric (5) is zero, but the corresponding dual metrics (14, 16, 17, 18) have non vanishing scalar curvatures and no RungeLenz vector. We found that the scalar curvature of dual metric (14) is positive and the corresponding scalar curvature for (17) is negative.

We have obtained that the dual metric (17) is a special case of extended Taub-NUT metric given in 15. We obtained also that all dual Taub-NUT metrics are not conformally flat. The spinning space was constructed and the generic and non-generic symmetries of the dual Taub-NUT metrics were analyzed. We found that dual Taub-NUT metrics have not non-generic symmetries. Our result differs from those presented in [3]. Geometric duality, in case of Taub-NUT metric, reduces the number of symmetries of the dual metrics. Finding the number of symmetries of dual metrics for a given manifold which admits a Killing tensor is an interesting problem, and it requires further investigation. 


\section{Acknowledgments:}

One of the authors (D.B.) thanks Prof.S.Bażański and Prof. S. Manoff, for helpful discussions and for continuous encouragements.

\section{References}

[1] R.H Rietdjik and J.W. van Holten, J. Geom. Phys. (1993)11 559.

[2] G.W. Gibbons, R.H Rietdijk and J.W. van Holten, Nucl. Phys.1993 B 40442.

[3] R.H. Rietdjik, J.W. van Holten 1996 Nucl. Phys. (B472) 472-446.

[4] S.L. Bażański,9th Italian Conference on General Relativity and Gravitational Physics, pp.2-9, R.Cianci et al..eds..World Scientific, Singapore, $(1991)$

[5] D.Baleanu,gr-qc/9805053

D. Baleanu, gr-qc/9805052

[6] D.J.Gross and M.J.Perry, Nucl.Phys. B226 (1983) 29.

[7] R.D.Sorkin, Phys.Rev.Lett. 51 (1983) 87.

[8] N.S.Manton, Phys.Lett. B110 (1982) 54; id, B154 (1985) 397; id, (E) B157 (1985) 475.

[9] M.F.Atiyah and N.Hitchin, Phys.Lett. A107 (1985) 21.

[10] G.W. Gibbons and P.J. Ruback, Phys. Lett. B188 (1987) 226; Commun. Math. Phys. 115 (1988) 267.

[11] G.W. Gibbons and N.S. Manton, Nucl. Phys. B274 (1986) 183.

[12] L.Gy. Feher and P.A. Horvathy, Phys. Lett. B182 (1987) 183; id, (E) B188 (1987) 512.

[13] B. Cordani, L.Gy. Feher and P.A. Horvathy, Phys. Lett. B201 (1988) 481.

[14] D.Baleanu, Helv.Phys.Acta 67 (1994)405

D.Baleanu, Il Nuovo Cimento B 109 (1994)845

D.Baleanu, Il Nuovo Cimento B 111(1996)973

M. Vişinescu and D. Vaman, Phys.Rev. D57(1998) 3790-3793.

[15] D. Baleanu, Gen. Rel. and Grav. 199830 no.2 195.

[16] D. Baleanu, Helv. Acta Phys. 199870 . 
[17] D. Baleanu, Non-generic Symmetries on Extended Taub-NUT metric Particles, Fields and Gravity, Lodz-Poland,(1998) 15-19 April

[18] J.W.van Holten, Phys.Lett.B 342(1995) 47-52 


\section{Appendix 1.}

For the metric (14) non-vanishing Christoffel coefficients are

$$
\begin{aligned}
& \Gamma_{11}^{1}=-\frac{m}{r(r+2 m)}, \quad \Gamma_{12}^{2}=-\frac{m^{2}+4 m r+2 r^{2}}{r\left(r^{2}+3 r m+2 m^{2}\right)} \\
& \Gamma_{13}^{3}=\frac{m^{2}+4 r m+2 r^{2}}{r\left(r^{2}+3 r m+2 m^{2}\right)}, \quad \Gamma_{13}^{4}=-\frac{\cos (\theta)(3 m+2 r)}{r^{2}+3 r m+2 m^{2}} \\
& \Gamma_{14}^{4}=\frac{m}{r(r+2 m)}, \quad \Gamma_{22}^{1}=-\frac{r(r+m)\left(m^{2}+4 r m+2 r^{2}\right)}{(r+2 m) m^{2}} \\
& \Gamma_{23}^{3}=\frac{\cos (\theta)\left(r^{4}+6 r^{3} m+13 r^{2} m^{2}+12 r m^{3}+2 m^{4}\right)}{\sin (\theta)\left(r^{4}+6 r^{3} m+13 r^{2} m^{2}+12 r m^{3}+4 m^{4}\right)} \\
& \Gamma_{23}^{4}=-\frac{\left(\cos ^{2}(\theta)+1\right)\left(r^{4}+6 m r^{3}+13 r^{2} m^{2}+12 r m^{3}\right)+4 m^{4}}{2 \sin (\theta)\left(r^{4}+6 r^{3} m+13 r^{2} m^{2}+12 r m^{3}+4 m^{4}\right)} \\
& \Gamma_{24}^{3}=-2 \frac{m^{4}}{\sin (\theta)\left(r^{4}+6 r^{3} m+13 r^{2} m^{2}+12 r m^{3}+4 m^{4}\right)} \\
& \Gamma_{24}^{4}=2 \frac{m^{4} \cos (\theta)}{\sin (\theta)\left(r^{4}+6 r^{3} m+13 r^{2} m^{2}+12 r m^{3}+4 m^{4}\right)} \\
& \Gamma_{33}^{1}=-\frac{r\left(2 r^{5} \sin ^{2}(\theta)+14 r^{4} m \sin ^{2}(\theta)+37 r^{3} m^{2} \sin ^{2}(\theta)+45 r^{2} m^{3} \sin ^{2}(\theta)+24 r m^{4} \sin ^{2}(\theta)+4 m^{5}\right)}{m^{2}\left(r^{3}+6 r^{2} m+12 r m^{2}+8 m^{3}\right)} \\
& \Gamma_{33}^{2}=-\frac{r \sin (\theta) \cos (\theta)\left(r^{3}+6 r^{2} m+13 r m^{2}+12 m^{3}\right)}{\sin (\theta)\left(r^{4}+6 r^{3} m+13 r^{2} m^{2}+12 r m^{3}+4 m^{4}\right)} \\
& \Gamma_{34}^{1}=-4 \frac{m^{3} r \cos (\theta)}{r^{3}+6 r^{2} m+12 r m^{2}+8 m^{3}} \\
& \Gamma_{34}^{2}=2 \frac{m^{4} \sin (\theta)}{r^{4}+6 r^{3} m+13 r^{2} m^{2}+12 r m^{3}+4 m^{4}}, \quad \Gamma_{44}^{1}=-4 \frac{m^{3} r}{(r+2 m)^{3}}
\end{aligned}
$$

and the curvature

$$
R=2 \frac{6 m^{3}+21 r m^{2}+22 r^{2} m+8 r^{3}}{2 m^{5}+9 r m^{4}+16 r^{2} m^{3}+14 m^{2} r^{3}+6 r^{4} m+r^{5}}
$$

For the metric (17) non-vanishing Christoffel components are

$$
\Gamma_{11}^{1}=-\frac{m}{r(r+2 m)}, \quad \Gamma_{12}^{2}=\frac{m^{2}}{r(r+m)(r+2 m)}
$$




$$
\begin{gathered}
\Gamma_{13}^{3}=\frac{m^{2}}{r\left(r^{2}+3 r m+2 m^{2}\right)}, \quad \Gamma_{13}^{4}=\frac{m \cos (\theta)}{r^{2}+3 r m+2 m^{2}}, \quad \Gamma_{14}^{4}=\frac{m}{r(r+2 m)} \\
\Gamma_{22}^{1}=-\frac{r m^{4}}{(r+m)^{3}(r+2 m)}, \quad \Gamma_{23}^{3}=-\frac{\left(r^{2}-2 m^{2}\right) \cos (\theta)}{\sin (\theta)(r+2 m)^{2}} \\
\Gamma_{23}^{4}=\frac{3 \cos ^{2}(\theta) r^{2}-r^{2}-4 \sin ^{2}(\theta) r m-4 m^{2}}{2 \sin (\theta)(r+2 m)^{2}} \\
\Gamma_{33}^{1}=-\frac{r m^{3}\left(r^{2} m+11 r^{2} m \cos ^{2}(\theta)+4 r m^{2}+8 r m^{2} \cos ^{2}(\theta)+4 m^{3}+4 r^{3} \cos ^{2}(\theta)\right)}{\left.r^{6}+9 r^{5} m+33 r^{4} m^{2}+63 r^{3} m^{3}+66 r^{2} m^{4}+36 r m^{5}+8 m^{6}\right)} \\
\Gamma_{33}^{3}=\frac{r(3 r+4 m) \cos (\theta) \sin ^{2}(\theta)}{\sin (\theta)(r+2 m)^{2}}, \quad \Gamma_{34}^{1}=-4 \frac{\left(r^{2}+2 r m+m^{2}\right.}{r^{3}+6 r^{2} m+12 r m^{2}+8 m^{3}} \\
\Gamma_{34}^{2}=2 \frac{(r+m)^{2} \sin (\theta)}{(r+2 m)^{2}}, \quad \Gamma_{44}^{1}=-4 \frac{m^{3} r}{(r+2 m)^{3}}
\end{gathered}
$$

and the curvature is:

$$
R=-2 \frac{6 m^{2}+3 r m+2 r^{2}}{m\left(r^{3}+4 r^{2} m+5 r m^{2}+2 m^{3}\right)}
$$




\section{Appendix 2.}

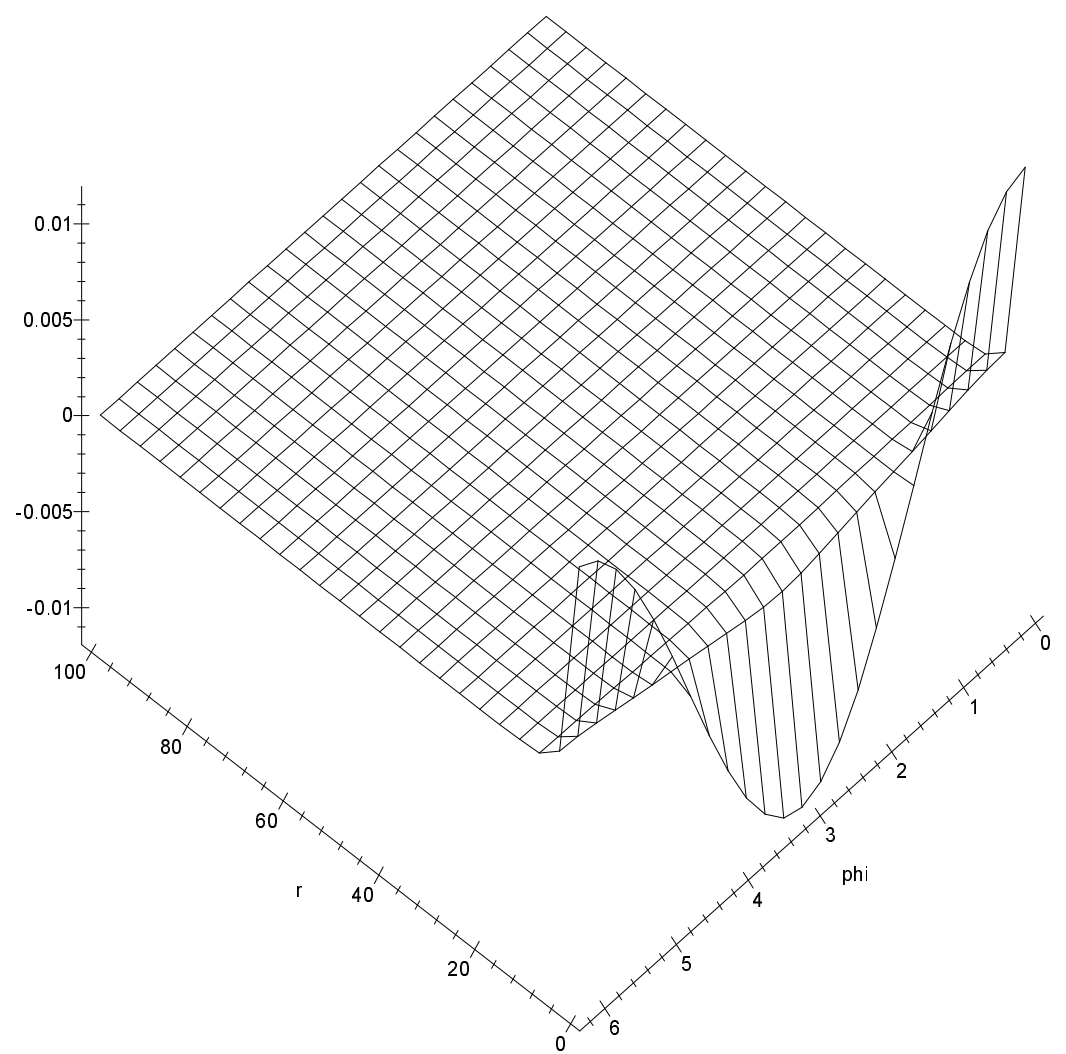

Figure 1: Ricciscalar corresponding to (16) for $i=1$ 


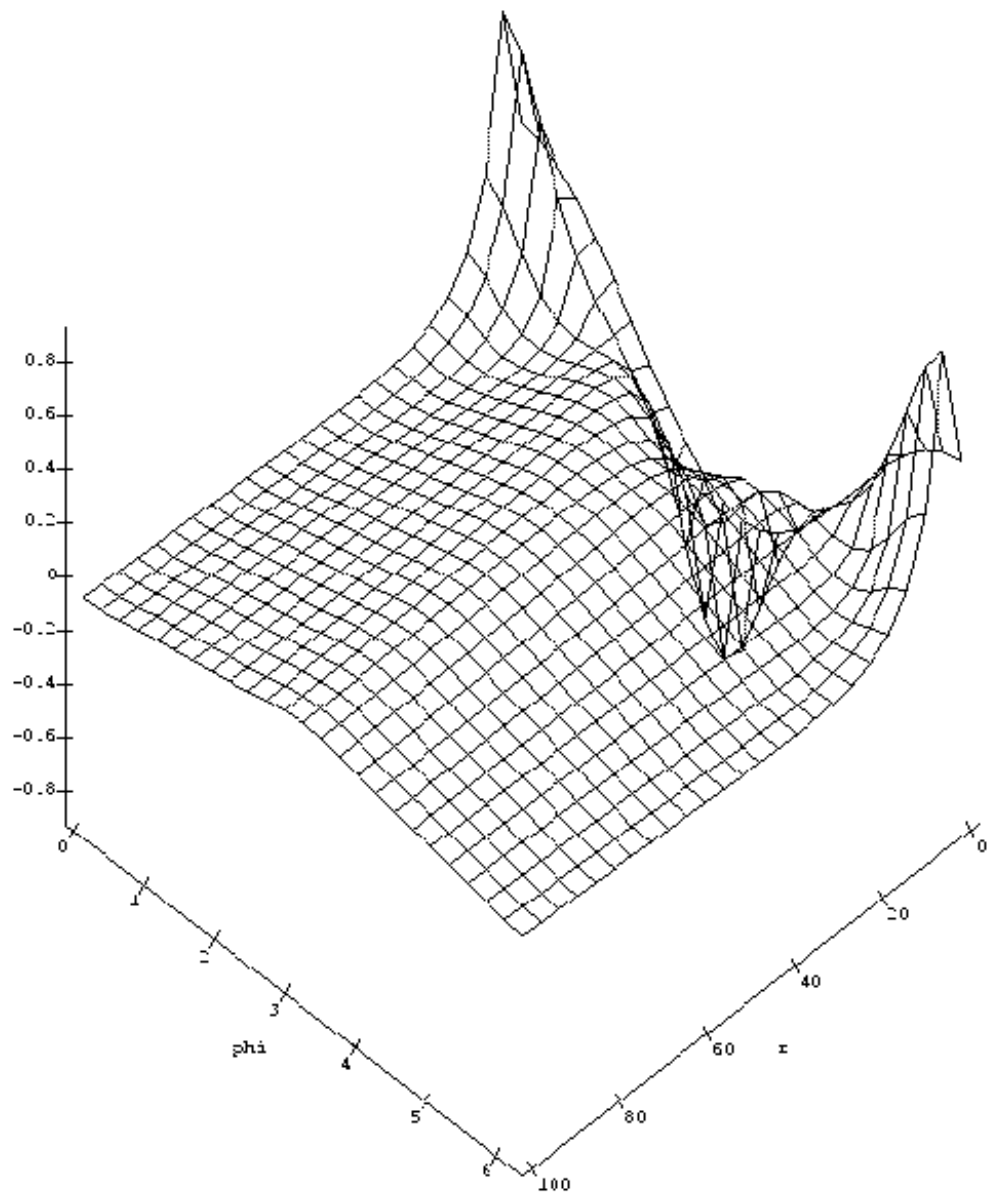

Figure 2: Ricciscalar corresponding to (18) for $\mathrm{i}=1$ 